\title{
Music Therapy Decreases Sadness and Increases Happiness in Alzheimer Patients: A Pilot Study
}

Jose Enrique de la Rubia Ortí1, , María Pilar García-Pardo ${ }^{2, \dagger}$, María Benlloch', Eraci Drehmer1, José Luis Platero', Alejandro Salazar ${ }^{3}$, David Sancho ${ }^{4}$, María Mar López-Rodríguez ${ }^{5+}$

\section{ABSTRACT}

\section{Background}

Alzheimer's disease (AD) is an irreversible neuro-degenerative dementia that causes emotional disorders such as anxiety and depression associated with low well-being and changes of state of mind. The aim of the present study was to assess the impact of a music therapy protocol as a tool to improve basic emotions in AD.

\section{Methods}

It is an analytical, experimental and prospective study. 30 Alzheimer patients of a mild level with an average age of 76,28 $\pm 4,6$ years, participated in the study. Participants were randomly assigned either to a Control Group $(n=15)$ or an Intervention Group $(n=15)$ that received music therapy twice a week during three months (24 sessions). Due to some limitations of the work, it is only a pilot study.

\section{Results}

The data showed that no significant differences exist between pre and post values in the control group (that did not receive music therapy) in any basic emotion measure (fear, happiness, anger and sadness). However, in the intervention group (that received music therapy for three months) a significant improvement can be observed in all the emotions comparing pre and post intervention (more happiness and less fear, anger and sadness). Moreover, the result comparing control and intervention group between them in post-session showed that the patients of the intervention groups had a significant improvement in happiness and sadness after the application of music therapy.

\section{Conclusions}

An intervention using music therapy increases the state of happiness and decreases sadness in mild Alzheimer sufferers.

\section{Keywords}

Alzheimer disease, Music therapy, Sadness, Happiness, Well-being

\footnotetext{
'Faculty of Nursing, Catholic University of Valencia, c/ Espartero, 7, 46007, Valencia, Spain

${ }^{2}$ Department of Psychology and Sociology, Faculty of Social and Human Sciences, University of Zaragoza, Spain

${ }^{3}$ Preventive Medicine and Public Health Area, University of Cádiz, Spain; The Observatory of Pain (External Chair of Pain), University of Cádiz, Spain; Institute of Research and Innovation in Biomedical Sciences of the Province of Cádiz, INiBICA, Spain

${ }^{4}$ Faculty of Psychology, Catholic University of Valencia, c/ Espartero, 7, 46007, Valencia, Spain

5Department of Nursing, Phisiotherpy and Medicine, University of Almería. La Cañada de San Urbano 04120, Almería, Spain

${ }^{\dagger}$ Author for correspondence: José Enrique de la Rubia Ortí, PhD Associate Professor, Faculty of Nursing, Catholic University of Valencia, c/ Espartero, 7, 46007, Valencia, Spain, email:joseenrique.delarubi@ucv.es

María Pilar GARCÍA PARDO, Department of Psychology and Sociology, Faculty of Social and Human Sciences, University of Zaragoza, Campus of Teruel, C/Atarazana, s/n. 44003 Teruel, Spain, email:magapar@unizar.es

María Mar López-Rodríguez, Department of Nursing, Phisiotherpy and Medicine, University of Almería. La Cañada de San Urbano 04120, Almería, Spain, email: mlr295@ual.es
} 


\section{Introduction}

Alzheimer's disease (AD) is an irreversible disorder with serious health, mental and social consequences in the patients during the development of the illness along the years. However, now it has no cure. The most relevant and known symptoms associated with this disorder are a decline in cognitive functions such as loss of memory, orientation task and poor attention. However, other behavior and mood pathologies have been associated. In this regard, anxiety and depression are typically described in patients with $\mathrm{AD}[1,2]$ inducing a lack of wellbeing [3]. It has also been described that patients with mild degree Alzheimer showed high levels of cortisol (the main hormone involved in stress situations) that induce a negative mood state $[4,5]$. Depression is one of the most common neuropsychiatric disorders in $\mathrm{AD}$ [6]. In fact, it is a higher considered risk factor strongly associated with the progression of the disease [7]. On the other hand, anxiety is more common in individuals with dementia than individuals without dementia, associated with a poor quality of life, behavioral problems and less neuropsychological performance [8]. Studies have also associated changes in the stress system with the appearance of mood pathologies, damage in the human hippocampus or risk to dementia development $[9,10]$. Moreover, in AD patients brain damage at the level of the limbic system linked to basic emotions have been described [11] inducing modifications of emotional expressions $[12,13]$. Therefore, one of the therapeutic strategies used in the treatment of Alzheimer should be to improve the frame of mind, through the improvement of basic emotions.

In this context, different non-pharmaceutical interventions have tried to improve a decrease in anxiety and depression [14] or the perception of wellbeing in $\mathrm{AD}$ [15]. For example, physical exercise using information and communication technology (ICTs) and anaerobic physical exercise have demonstrated that they decrease levels of negative mood [16]. Within these nonpharmacological interventions, music therapy has showed wide results in different disorders $[17,18]$ highlighting as being able to modulate different factors related to mood state $[18,19]$.

Concretely, regarding with cognitive damage patients it was particularly efficient because it induces the cerebral plasticity at the beginning of disorders, for example, for Alzheimer sufferers [20]. Music therapy not only helps AD patients cope with their negative emotions, it can also promote wellness [15], express feelings [21,22] or it led to positive emotional changes $[23,24]$. Moreover, it improved irritability, social insolation and anxiety and depression symtoms [25]. It has even been demonstrated that only a short-intervention of music therapy is enough to improve anxiety and depression levels in $\mathrm{AD}$ patients [4].

However, the study of the concrete emotions that improve after the intervention with music therapy is unknown. Some basic emotions are crucial in our internal homeostasis and the development of our life. These emotions permit us to survive and communicate with others. For example, happiness, sadness, fear and anger are essential in our lives and it is known that these emotions prosecution are modified by progressive neuropathological changes in the temporal and frontal areas may affect emotional processing in advanced stages of AD [26]. Based on this reasoning, the aim of our work is to study the effects of music intervention in patients with mild $\mathrm{AD}$ evaluating concretely the possible improvement in four essential and basic emotions (happiness, sadness, fear and anger) after the application of this therapy. Our hypothesis is that patients in the intervention group will show high punctuation in basic emotions regarding positive emotional state (happiness) and low punctuations in basic emotions regarding negative emotional state (sadness, fear and anger) that patients without music intervention. Based in our small sample size our work is only a pilot study.

\section{Methods \\ - Study design}

It is an analytical, experimental and prospective study.

\section{- Participants}

The participants in this study were mild Alzheimer sufferers belonging to the Family Association of Alzheimer sufferers of Valencia, Spain (FAAV), where the study was performed. The inclusion criteria that was applied was that patients had to be older than 65 years old with consent given by their legal tutors, affected by dementia type Alzheimer with a mild level of severity according to Mini Mental State of Examinations (MMSE) which corresponds with a score between 20-25 points in this test [27]. It was carried out before 
starting music therapy, by the clinic neurologist. Exclusion criteria were referred to patients who refused to participate in the study or did not meet the criteria inclusion. The required sample size was determined based on previous studies $[4,22]$ to detect a difference between groups of 4,55 points in the Mood State Questionnaire (MOOD test), with expected standard deviation of 5,04 and 2,95 in each group, respectively, a statistical power set to $80 \%$, and a confidence level of $95 \%$. Under these conditions, the necessary sample size was 13 patients in each group. However, the final sample was made up of 30 patients $(n=30)$ with an average age of 76,28 \pm 4,6 years and 22 points in the Mini Mental State Examination (MMSE) of 22 (Table 2). This sample was randomly distributed into two groups: 15 patients received music therapy during three months (the Intervention Group) and the other 15 patients formed the group that did not receive music therapy (nor any other type of intervention) during the three months of the study (Control Group) (Table 1). The gender of the patients was divided into $70 \%$ women and $30 \%$ men (Table 2), reproducing the percentages of the distribution of the illness according to gender [28]. The education level was similar in all patients (all of them possessed superior studies) and they all had a high social- economic level. As for the medication received, all of the patients took comparable doses of anticholynesterasics and none of the participants received drugs to decrease anxiety or depression over the 3 month period of intervention.

\section{- Procedure}

Just before starting the music therapy sessions the clinic psychologists carried out at the same time (9.15 a.m.), the evaluation of the emotional state of each subject was measured by the MOOD test (described in detail later). Simultaneously, and to avoid that the interpretation of the influence of music therapy could be biased, the level of anxiety and depression of both groups was compared using HADS (described in detail later) by the same psychologist using the two subscales that compose it.
The objective was to demonstrate that all patients in the study started with the same levels of anxiety and depression before the intervention with music therapy because the mood state could influence the basic emotions measures. In both cases (MOOD test and HADS test), the psychologists did not know which group (intervention or control) each patient belonged to. It could be observed that there were no differences between the two groups before the intervention in any of the variables studied ( $p>0.05)$ (Table 2).

Also, the values of cortisol (hormone associated with stress levels) were measured before to start the intervention with music therapy. It was obtained from each patient a sample of hair (100 strands of hair of at least $3 \mathrm{~cm}$ in length) taken using fine scissors from the base of the nape with the aim of demonstrating that the stress levels in the patients of both groups before beginning the music therapy sessions were similar too (Table 2).

To follow the professionals implemented the music therapy protocol to the 15 patients of the Intervention Group. These sessions were carried out in groups of five (in separate rooms) to increase the efficiency of the intervention, using the same music therapist with the same group until the end of the study. The sessions were continued during three months, Mondays and Thursdays of each week (a total of 24 sessions with a duration of 60 minutes through two activities which lasted 30 minutes each at the same time (10:00 a.m) and in a relaxed atmosphere (Table 1; Annex 1). While the sessions were taking place, the 15 patients of the Control Group were in rooms in the same center with the same conditions as the Intervention Group without receiving no type of therapy. These patients were in a relaxed environment in their room interacting with each other without performing any specific task. All the patients (both the control group and the intervention group) attended all the sessions. After each therapy session was concluded, the participants of each group (intervention and control) were once again subjected to the MOOD test at the same time (11.00 a.m.) (Table 1 ) and by

\begin{tabular}{|l|l|l|l|l|l|}
\hline Table 1: Distribution of therapies and interviews during the $\mathbf{1 2}$ weeks of the study. \\
\hline & N & Day of week & $\begin{array}{l}9: 15 \\
\text { a.m }\end{array}$ & 10:00 a.m & 11:00 a.m \\
\hline Intervention Group & 15 & Monday and Thursday & MOOD & Music-therapy Session & MOOD \\
\hline Control Group & 15 & Monday and Thursday & MOOD & No therapy & MOOD \\
\hline
\end{tabular}


Research José Enrique de la Rubia Ortí

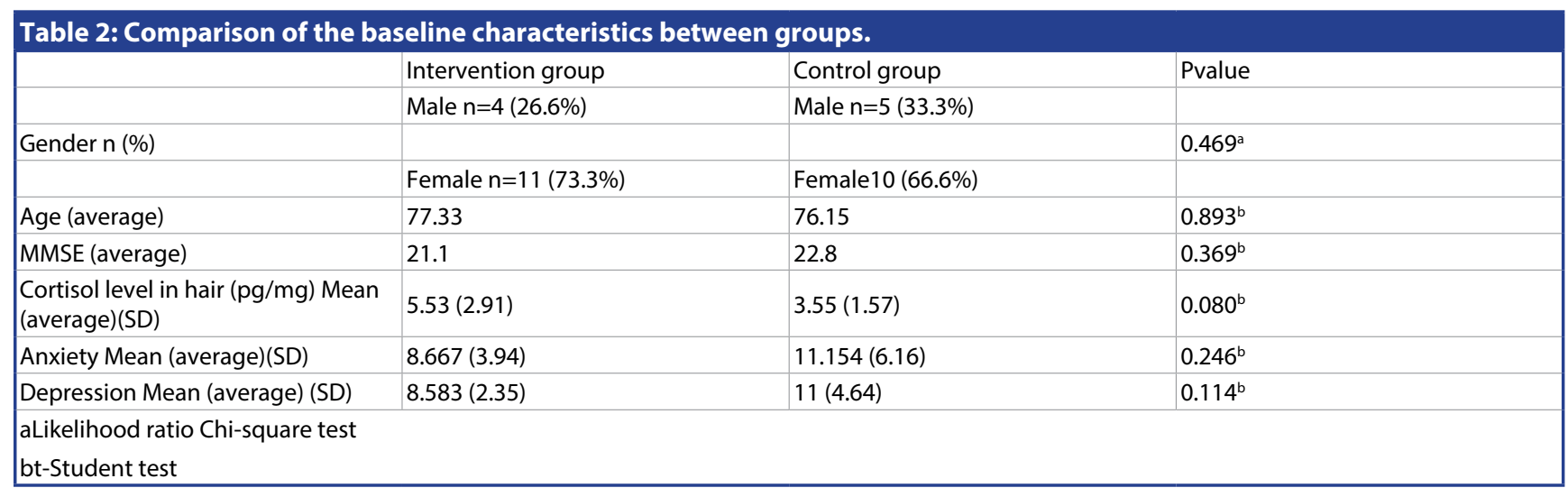

the same neurologist who was assigned before intervention, obtaining the records of the tests of all the participants of the study.

\section{- Annex 1: Protocol of the music therapy session}

Activity 1: The welcome song whose goal was to activate the cognitive area in order to improve recent memory such as remembering the names of classmates, the lyrics of the song, visual agnosia with the recognition of faces, attention with concentration on the musical task, orientation with the recognition of bandmates and recognition of the present day of the week (session day), and to also activate the socialemotional area in order to improve self-esteem from a successful experience and to participate in a vocal group activity on a verbal level, interacting with peers and the music therapist.

Activity 2: Theme song related to flowers in order to improve their cognitive level and memory, remembering the lyrics of the songs and the songs used in the session, the language for singing the lyrics and saying the name of the flowers and/or plants that were mentioned in the songs, focusing attention on the musical task and temporal orientation linking flowers with the spring season. The activity also pursued to improve their socio-emotional level, which seeks to improve self-esteem from a successful experience, to participate in a vocal group activity, to verbally interact with peers and the music therapist, to express feelings and express opinions and/or musical preferences. Finally, on a physical-motor level, the song aimed to stimulate the respiratory functions.

\section{- Instruments}

To evaluate the change of moods of the study patients a Spanish adaptation of the MOOD test was used (Mood State Questionnaire) [29]. This test consists of a self-report measure and it groups equally positive and negative states of mind. It consists of 20 questions with 3 possible answers ( $1=$ never, $2=$ sometimes, $3=$ often). These 20 questions were grouped in 4 states of well-being: happiness, anger, sadness and fear, getting a global vision of the individual. The score of each emotional state was calculated as the average score of the items in that specific dimension. The questionnaire gave a satisfactory validity and a credibility of 0,85 and it has already been used in patients with Alzheimer in this same state of affectation showing good results [15].

The test Hospital Anxiety and Depression Scale (HADS) [30-32] is a self-report test composed by 14 items and two subscales (subscale of depression and subscale of anxiety with seven items each) with format of Likert scale 0-3 and with a punctuation range in each subscale of 0-21. Higher score indicates greater anxiety and depression in both subscales. Normally, it has been described that a score greater tan eleven would indicate "case" and lower than eight would be considered "probable case" [31,33]. Despite being a self- report scale which is very simple, with few questions, and for patients who are still aware of their emotions, it is considered appropriate for this study [34]. The scale has high internal consistency (alpha Cronbach of 0.7-0.9) and high stability $(r>0.80)$ [30].

For the quantification of cortisol, each hair sample was incubated with $1 \mathrm{ml}$ of methanol at a temperature of $34^{\circ} \mathrm{C}$, shaken slightly for 36 hours, and below we quantify the cortisol in the hair samples by Enzyme-Linked Immunosorbent (ELISA) [35] using the Salivary Cortisol ELISA SLV-2930 kit (DRG International Inc.).

\section{- Data analysis}

The potential baseline differences between the control group and the intervention group were 
checked via chi-square test (in the case of gender) and $\mathrm{t}$-Student tests (rest of the cases), after checking for normality (Kolmogorov-Smirnov test). A descriptive analysis of the baseline scores and post-scores of the 4 emotional dimensions was carried out, reporting means and standard deviations. In addition, the changes in the 4 dimensions were also analyzed. For this purpose, four new variables were created, namely "change in happiness", "change in anger", "change in sadness" and "change in fear". Each of these variables was calculated as the difference of posttreatment scores and pre- treatment scores of the MOOD.

On the other hand, we analysed the baseline and post-scores differences, as well as the differences in the aforementioned changes along time within groups and between groups using paired $\mathrm{t}$-Student tests and independent $\mathrm{t}$-Student tests, respectively, after checking for normality (Kolmogorov-Smirnov test).

\section{- Ethical considerations}

We considered all of the basic principles of biomedical research described in the Helsinki declaration, with the approval of the research ethics committee of the Commission on Human Ethics in Experimental research of the University of Valencia on June $29^{\text {th }}, 2015$.

\section{Results}

The mean scores of each dimension (pre, post, and changes over time) in each group after the three months of the intervention are presented in
Table 3. In the control group, pre-intervention scores were $2.09 \pm 0.46$ for fear, $2.09 \pm 0.54$ for happiness, $2.03 \pm 0.40$ for anger and finally $2.03 \pm 0.65$ for sadness. Meanwhile, after (post measures), the values were $2.07 \pm 0.32,1.86 \pm$ $0.57,2.19 \pm 0.35$, and $2.11 \pm 0.49$, respectively, not finding any significant changes (post-pre) for any dimension in control group. (Table 3 and Figure 1A). Nevertheless, in the intervention group, the pre-intervention scores were 2.20 \pm 0.20 for fear, $2 \pm 0.33$ for happiness, $2.31 \pm$ 0.51 for anger, and $2.27 \pm 0.32$ for sadness. The scores on post-intervention after the period, which music therapy were $1.83 \pm 0.28,2.37 \pm$ $0.56,1.85 \pm 0.58$ and $1.64 \pm 0.53$ respectively, finding significant changes $(\mathrm{p}<0.05)$ in fear, anger, sadness, and happiness (Table 3 and Figure 1B).

Moreover, a comparative between the two groups was also performed and the end of the intervention (three months). The results of the comparisons between the groups showed no significant baseline differences (pre-scores) between intervention and control group (Table 3). However, regarding the post-measures, we observed significant differences in happiness and sadness between intervention and control groups, showing the intervention group more happiness (2.37 vs. $1.86 ; \mathrm{p}=0.036)$ and less sadness $(1.64$ vs. 2.11; $\mathrm{p}=0.033$ ) (Table 3 and Figure 1B).

\section{Discussion}

This study has demonstrated for the first time that music therapy induces a change in basic

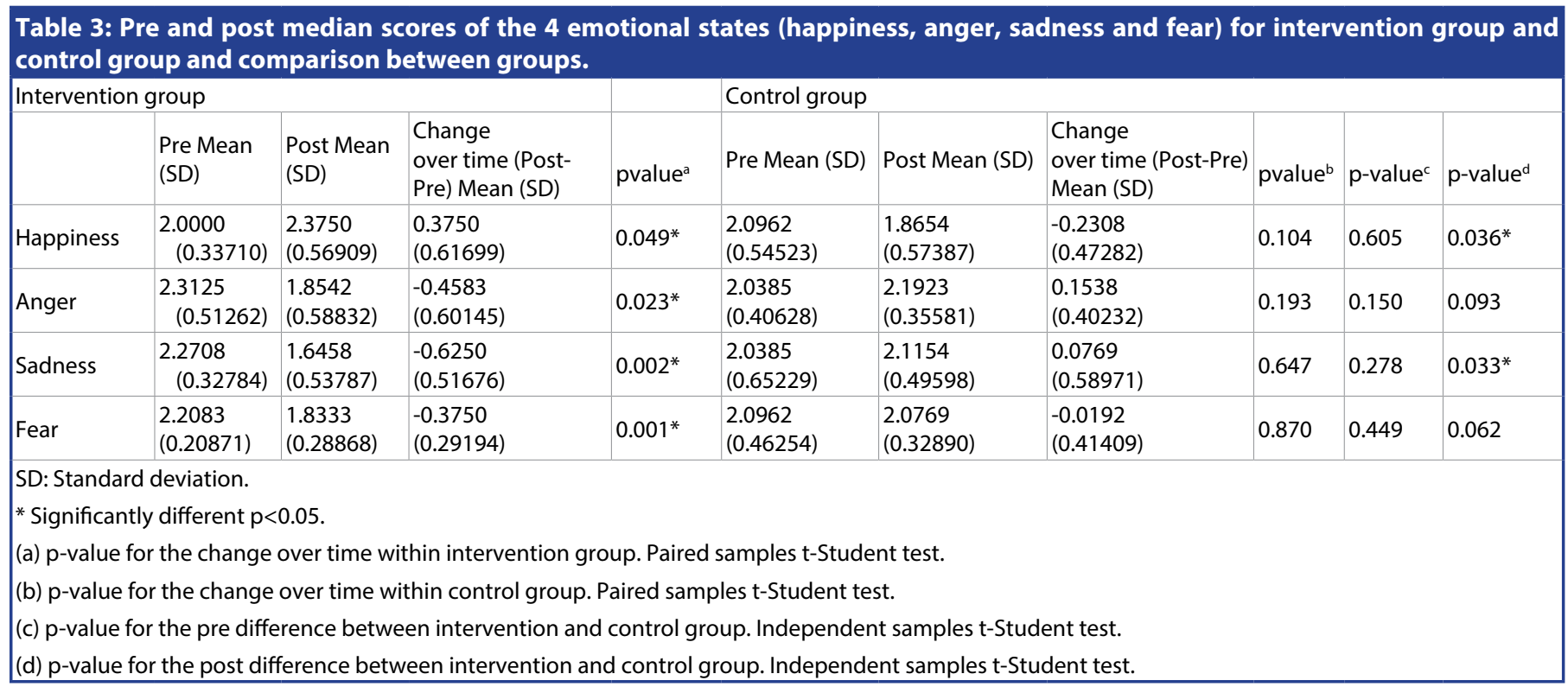




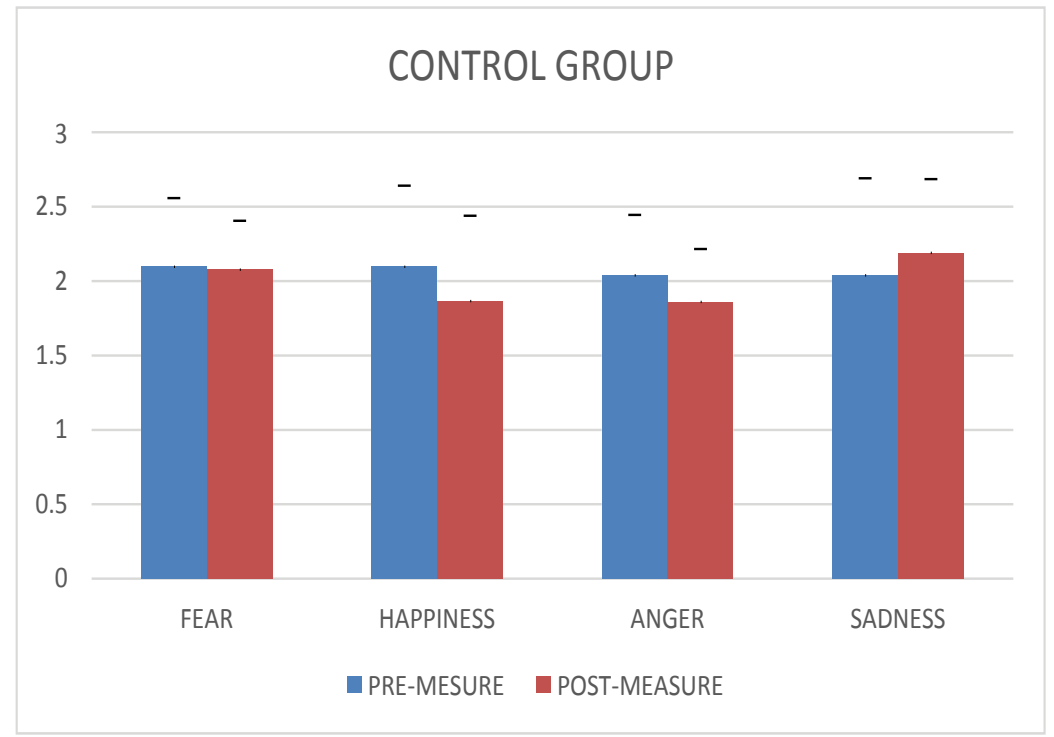

MUSIC THERAPY INTERVENTION GROUP

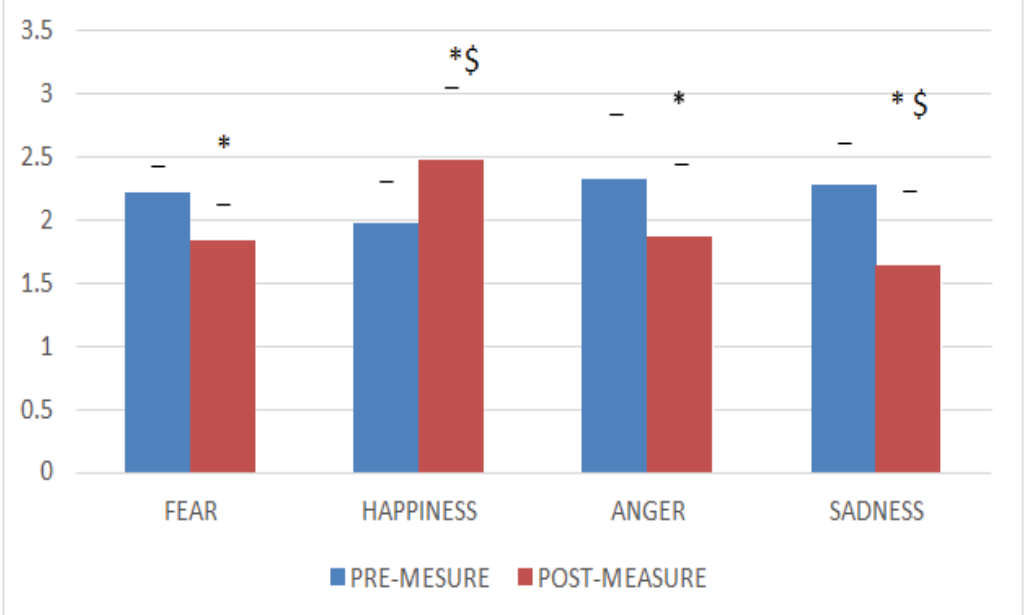

Figure 1: Comparative Table of the scores of fear, happiness, anger and sadness (obtained after applying the MOOD Test in Control Group who not received music therapy and in Intervention Group).

Note: ${ }^{*}<0.05$ significant differences between pre and post-measure; ${ }^{*}>0.05$ significant differences between control and music intervention group

emotions (happiness, sadness, fear and anger) in $\mathrm{AD}$ patients. Experience of this type of therapy increases the expression of happiness and decreases sadness, fear and anger. In addition, we have demonstrated that patients that experience music therapy compared with patients that did not, increase their mood state expressing more happiness and less sadness.

These results are in agreement with a previous work from our laboratory that demonstrated that exposure to a single and acute session of music therapy reduces anxiety and depression levels in $\mathrm{AD}$ patients [4]. We also observed that long-term music therapy increases the perception of wellbeing (happiness) [15]. From our point of view, the improvement of positive emotions and the decrease of negative state after this type of therapy could be due to the fact that music therapy is able to activate the cortical area (especially the orbitofrontal cortex, the superior temporal cortex and the anterior cingulate $[36,37]$ and subcortical areas, such as, the limbic and para-limbic areas [38] highlighting the parahipocampal and amygdala [39] that are areas involved of emotional state in $\mathrm{AD}$ patients [1].

According to our study the variables happiness and sadness in the intervention group showed significant improvements after the treatment was applied compared to the control group that did not receive this type of therapy. The fact that only these two emotions improved significantly after stimulation by musical therapy, could be explained by the hypthotesis described in other studies showing that after using musical therapy as stimulation it produces an asymmetrical pattern in the superior temporal cortex areas associated with these two emotions [40]. In this sense, new theories point out that despite emotions being related fundamentally to the sub cortical regions located in the limbic system (highlighting the amygdala), in reality they depend on multiple cerebral networks in which they connect cortical and subcortical structures so establishing function patterns for each of the basic emotions [41]. This fact could explain that the brain damage that occurs with Alzheimers sufferers does not affect all of the emotions in the same manner, which shows that the improvement produced from whichever type of therapy will not be of the same intensity for each one of them. Confirming this reasoning, non pharmalogical therapies applied to Alzheimers sufferers like stimuli film clips managed to improve Alzheimer patients precisely in sadness and happiness [42] coinciding with the results obtained in our study. This made us think that the neural networks that depend on both emotions could be related.

To try and explain these emotional changes, other authors have established a hypothesis that directly relates stress with the perception of happiness/sadness. It seems that happiness/ sadness emotional processing might be related to stress reduction by music [40]. So, delving into the mechanism of why music therapy is able to achieve an improvement in these two specific variables, the level decrease of cortisol could be the explanation, because it decreases after 
applying a single therapy, existing a correlation between an improvement in stress levels and a perception of anxiety and stress [4]. Specifically the perception of happiness has already been related by other authors with levels of cortisol [43] and in relation to the perception of sadness, it can also be observed how it increased in stressful situations [44]. Therefore, our results show an improvement in the well-being of patients in line with other published results after music therapy $[15,23]$. Concretely, the treatment of our study was applied during 3 months confirming the emotional improvement observed during only one session [4] and affirming that music therapy has a positive effect in both case (acute and longterm). In this direction, it is known that despite the damage in the hippocampus in Alzheimers patients and damages declarative memory (so that the stimuli used to achieve an improved emotional state can be forgotten) [45] the memory of emotions depends on implicit and emotional memory not strongly affected in $\mathrm{AD}$ patients. In this regard, [13] the amygdala is important for regulating and sustaining an emotion independent of hippocampal function and declarative memory for the emotion- inducing event. This could explain why the appliance of music therapy in a long period of time has a maintained impact in improving the patient, proving to be important in the evolution of the illness and improving the expression of the emotional state.

In conclusion, the present study demonstrated for first time that music therapy intervention applied over 3 months improves emotional state of mild Alzheimers patients producing a significant change concretely in sadness and happiness emotions.

Finally although our results are significant, this study has its limitations, highlighting the small sample used and will be recommended to propose an intervention with a larger group of patients. For this reason, it is only a pilot study.

On the other hand, it would be important to do the therapy over a longer period of time, analyzing at the same time the impact that music therapy produces at cerebral level, involving structural changes that could explain the maintenance in improvement time, associating each variable with different modified regions.

\section{Acknowledgements}

We are grateful to all of the participants in the study for their collaboration in this project. We would particularly like to thank the AFAV association for their collaboration in this study, providing us with the places and professionals to collect the data.

\section{References}

1. Suárez-González A, Crutch SJ, Franco-Macías $E$, et al. Neuropsychiatric symptoms in Posterior cortical atrophy and Alzheimer disease. $J$. geriat. Psychiatry. Neurol 29(2), 65-71 (2016).

2. García-Alberca JM. Cognitive-behavioral treatment for depressed patients with Alzheimer's disease. An open trial. Arch. Gerontol. geriat 71(1), 1-8 (2017).

3. Black DS, Slavich GM. Mindfulness meditation and the immune system: a systematic review of randomized controlled trials. Ann. N. Y. Acad. Sci 1373(1), 13-24 (2016).

4. De la Rubia Ortí JE, García-Pardo MP, Cabañés Iranzo C, et al. Does Music Therapy Improve Anxiety and Depression in Alzheimer's Patients?. J. Altern. Complement. Med 24 (1) 33-36 (2017)

5. De la Rubia Ortí JE, Castillo S, Benlloch SM et al. Impact of the Relationship of Stress and the Immune System in the Appearance of Alzheimer's Disease. J. Alzheimers. Dis 55(3), 899-903 (2016).

6. Wuwongse S, Chang RCC, Law AC. The putative neurodegenerative links between depression and Alzheimer's disease. Prog. neurobiol 91(4), 362-375 (2010).
7. Li JQ, Tan L, Wang HF, et al. Risk factors for predicting progression from mild cognitive impairment to Alzheimer's disease: a systematic review and meta- analysis of cohort studies. J. Neurol. Neurosur. Psychiatry 87(5), 476-484 (2016).

8. Seignourel PJ, Kunik ME, Snow L, et al. Anxiety in dementia: a critical review. Clin. Psychol. Rev 28(7), 1071-1082 (2008).

9. Swaab DF, Bao AM, Lucassen PJ. The stress system in the human brain in depression and neurodegeneration. Ageing. Res. $\operatorname{Rev} 4(2), 141$ 194 (2005).

10. Zvěřová $M$, Fišar Z, Jirák $R$, et al. Plasma cortisol in Alzheimer's disease with or without depressive symptoms. Med. Sci. Monit 19 (10), 681-689 (2013).

11. Talarovicova A, Krskova L, Kiss A. Some assessments of the amygdala role in suprahypothalamic neuroendocrine regulation: a mini review. Endocr. Regul 41(4), 155-162 (2007).

12. Desmarais $P$, Lanctôt $K L$, Masellis $M$, et al. Social inappropriateness in neurodegenerative disorders. Int. Psychogeriatr 30(2), 197-207 (2018).

13. Guzmán-Vélez E, Warren DE, Feinstein JS, et al. Dissociable contributions of amygdala and hippocampus to emotion and memory in patients with Alzheimer's disease. Hippocampus 26(6), 727-738 (2016).

14. Ahmedani BK, Belville-Robertson T, Hirsch A et al. An Online Mental Health and Wellness Intervention Supplementing Standard Care of Depression and Anxiety. Arch. Psychiatr. Nurs 30(6), 666-670 (2016).

15. De la Rubia Ortí JE, García- Pardo MP, PérezRos $P$, et al. Iga and Alpha-Amylase: New Targets for Well-Being in Alzheimer's Disease Patients, a Pilot Study with Music Therapy. Neuropsychiatry. (London) 8(1) 378-383 (2018).

16. De la Rubia Orti JE, Iranzo CC, Platero Armero $\mathrm{JL}$, et al. Impact on Information and Communication Technologies versus anaerobic physical activity in patients with mild Alzheimer' s disease: A pilot study. Neuropsychiatry. (London) 7(1), 37-41 (2017).

17. Mössler K, Gold C, Aßmus J et al. The therapeutic relationship as predictor of change in music therapy with young children with autism spectrum disorder. J. Autism. Dev. Disord 1-15 (2017).

18. Schneider MA. The Effect of Listening to Music on Postoperative Pain in Adult Orthopedic Patients. J. Holist. Nurs 36(1), 23-32 (2016). 
19. Lee WP, Wu PY, Lee MY, et al. Music listening alleviates anxiety and physiological responses in patients receiving spinal anesthesia. Complement. Ther. Med 31(1), 8-13 (2017).

20. Herholz SC, Herholz RS, Herholz K. Non-pharmacological interventions and neuroplasticity in early stage Alzheimer's disease, Expert. Rev. Neurother 13(11), 1235 1245 (2013).

21. MM Stanczyk. Music therapy in supportive cancer care. Rep. Pract. Oncol. Radiother 16(5), 170-172 (2011).

22. Suzuki M, Kanamori M, Watanabe $M$, et al. Behavioral and endocrinological evaluation of music therapy for elderly patients with dementia. Nur. Health. Sci 6(1), 11-18 (2004).

23. Narme $P$, Clément $S$, Ehrlé $N$, et al. Efficacy of musical interventions in dementia: evidence from a randomized controlled trial. $J$. Alzheimers. Dis 38(2), 359-369 (2014).

24. Onieva-Zafra MD, Hernández-Garcia L, Gonzalez-del-Valle MT, et al. Music Intervention With Reminiscence Therapy and Reality Orientation for Elderly People With Alzheimer Disease Living in a Nursing Home: A Pilot Study. Holist. Nurs. Pract 32(1), 43-50 (2018).

25. Raglio A, Bellelli G, Traficante D, et al. Efficacy of music therapy in the treatment of behavioral and psychiatric symptoms of dementia. Alzheimer. Dis. Assoc. Disord 22(2), 158-162 (2008).

26. García-Rodríguez B, Fusari A, Rodríguez $B$, et al. Differential patterns of implicit emotional processing in Alzheimer's disease and healthy aging. J. Alzheimers. Dis 18(3), 541-551 (2009).

27. Folstein MF, Folstein SE, McHugh, PR. Mini-mental state: a practical method for grading the cognitive state of patients for the clinician. J. psychiatric. Res 12(3), 189-
198 (1975).

28. Webber KM, Casadesus G, Perry G, et al. Gender differences in Alzheimer disease: the role of luteinizing hormone in disease pathogenesis. Alzheimer. Dis. Assoc. Disord 19(2), 95-99 (2005).

29. Górriz Plumed AB, Prado-Gascó VJ, Villanueva Badenes $L$, et al. The MOOD questionnaire: Adaptation and validation of the Spanish version. Psicothema 25(2), 480 (2013).

30. Samaras N, Herrmann FR, Samaras D, et al. The Hospital Anxiety and Depression Scale: low sensitivity for depression screening in demented and non- demented hospitalized elderly. Int. psychogeriatrics 25(1), 82-87 (2013).

31. Zigmond AS, Snaith RP. The hospital anxiety and depression scale. Acta. Psychiatr. Scand 67(6), 361-370 (1983).

32. Terol-Cantero MC, Cabrera-Perona V, Martín-Aragón M. Hospital Anxiety and Depression Scale (HADS) review in Spanish Samples. Ann. Psychology 31(2), 494-503 (2015).

33. Cabrera V, Martín-Aragón M, Terol MDC, et al. La Escala de Ansiedad y Depresión Hospitalaria (HAD) en fibromialgia: Análisis de sensibilidad y especificidad. Ter. psicol 33(3), 181-193 (2015).

34. Degirmenci $E$, Degirmenci T, Dügüncü $Y$, et al. Cognitive insight in Alzheimer's disease. Am. J. Alzheimers. Dis. Deme 28(3), 263-268 (2013).

35. Tecles F, Fuentes-Rubio M, Tvarijonaviciute $A$, et al. Assessment of stress associated with an oral public speech in veterinary students by salivary biomarkers. J. veter. Med. Edu 41(1), 37-43 (2014).

36. Blood AJ, Zatorre RJ. Intensely pleasurable responses to music correlate with activity in brain regions implicated in reward and emotion. Proc. Nat. Acad. Sci 98(20), 11818 11823 (2001).

37. Mistridis P, Taylor Kl, Kissler J, et al. Distinct neural systems underlying reduced emotional enhancement for positive and negative stimuli in early Alzheimer's disease. Front. Human. neurosci 20(7), 939 (2014).

38. Peretz I, Gagnon L, Bouchard B. Music and emotion: perceptual determinants, immediacy, and isolation after brain damage, Cognition 68(2), 111-141 (1998).

39. Gosselin N, Peretz I, Johnsen E, et al. Amyg dala damage impairs emotion recognition from music. Neuropsychologia 45(2), 236244 (2007).

40. Suda M, Morimoto K, Obata A, et al. Emotional responses to music: towards scientific perspectives on music therapy. Neurorep 19(1), 75-78 (2008).

41. Wager TD, Kang J, Johnson TD, et al. A Bayesian model of category-specific emotional brain responses. PLoSone 11(4), e1004066 (2015).

42. Mossello E, Ridolfi A, Mello AM, et al. Animal-assisted activity and emotional status of patients with Alzheimer's disease in day care. Int. Psychogeriatr 23(6), 899-905 (2011).

43. Burke HM, Davis MC, Otte C, et al. Depression and cortisol responses to psychological stress: a meta-analysis. Psychoneuroendocrinology 30(9), 846-856 (2005).

44. Mossink JC, Verkuil B, Burger AM, et al. Ambulatory assessed implicit affect is associated with salivary cortisol. Front. Psychology 10(6), 111 (2015).

45. Guzmán-Vélez E, Feinstein JS, Tranel D. Feelings without memory in Alzheimer disease. Cogn. Behav. Neurol 27(3), 117-129 (2014). 